\title{
On a Conjecture Concerning Positive Semi-definiteness
}

\author{
J. Recasens \\ Dept. Tecnologia de l'Arquitectura \\ Universitat Politècnica de Catalunya \\ Spain \\ j.recasens@upc. edu
}

\begin{abstract}
In [7] a conjecture relating the positive definiteness of a similarity with its transitivity with respect to the Eukasiewicz t-norm is made. In its current form, the conjecture is not true but from a modified version interesting consequences can be derived.

Keywords: Positive definite matrix, similarity, transitivity.
\end{abstract}

\section{Introduction}

In the paper [7] published in this journal an interesting Conjecture is presented concerning the positive definiteness of some similarities very much related to Fuzzy Logic [11] and especially to the theory of indistinguishability operators [8]. This Conjecture is not true in its current form as will be stated in the next section but in Section 3 a reformulation leading to interesting consequences is stated and proved.

Let us recall the definition of similarity and the conjecture presented in $[7]$.

Definition 1.1. [7] Let $E$ be a finite set and let $P(E)$ be its power set. A similarity is a mapping s from $P(E) \times P(E)$ into $\mathbb{R}^{+}$such that

a) $s(X, Y)=s(Y, X)$ for all $X, Y \in P(E)$ 
b) $s(X, Y) \leq s(X, X)$ for all $X, Y \in P(E)$.

A similarity $s$ gives rise to a matrix $S=\left(s\left(A_{i}, A_{j}\right)\right)$ that is called a similarity matrix in [7].

Conjecture 1.2. [7] Let $s: P(E) \times P(E) \rightarrow \mathbb{R}^{+}$be a similarity such that $s(X, X)=k$ for all $X \in P(E)$ and $s(X, Y)+s(Y, Z) \leq s(X, Z)+k$ for all $X, Y, Z \in P(E)$. Then the corresponding similarity matrix $S$ is positive semi-definite.

\section{Counterexample and Comments}

First of all let us notice that $A$ is a positive semi-definite matrix if and only if $p \cdot A$ is positive semi-definite for all $p>0$. So that dividing the matrix $S$ by $k$ in 1.2 we can assume that $k=1$ (i.e., it is reflexive) and that $s$ is valued in $[0,1]$. Then the condition of Conjecture 1.2 can be rewritten as

$$
\max (s(X, Y)+s(Y, Z)-1,0) \leq s(X, Z)
$$

Definition 2.1. [5] The operation $E:[0,1] \times[0,1] \rightarrow[0,1]$ defined for all $x, y \in[0,1]$ by

$$
E(x, y)=\max (x+y-1,0)
$$

is called the Eukasiewicz t-norm.

Definition 2.2. Given a set $X$, a similarity $s: X \times X \rightarrow[0,1]$ is E-transitive if for all $x, y, z \in X$,

$$
E(s(x, y), s(y, z)) \leq s(x, z) .
$$

A generalization of Conjecture 1.2 to finite sets of any cardinality is then:

Conjecture 2.3. If a reflexive similarity $s: X \times X \rightarrow[0,1]$ on a finite set $X$ is E-transitive, then its corresponding similarity matrix $S$ is positive semi-definite.

The next counterexample shows that the conjecture fails for sets of cardinality greater than or equal to 5 . 
Counterexample 2.4. The similarity with matrix

$$
S=\left(\begin{array}{ccccc}
1 & 0.4 & 0.6 & 0.2 & 0.8 \\
0.4 & 1 & 0.8 & 0.4 & 0.6 \\
0.6 & 0.8 & 1 & 0.6 & 0.4 \\
0.2 & 0.4 & 0.6 & 1 & 0.4 \\
0.8 & 0.6 & 0.4 & 0.4 & 1
\end{array}\right)
$$

is reflexive and E-transitive but its determinant is -0.03584 and one of its eigenvalues is -0.0512922301693901 .

The reason for this comes from the following results.

Definition 2.5. If a metric space $(S, d)$ is isometrically embeddable in an Euclidean space, we will say that $d$ is Euclidean.

Proposition 2.6. [9] Let $(S, d), S=\left\{x_{0}, x_{1}, \ldots, x_{n}\right\}$, be a finite metric space of $n+1$ points. Then $d$ is Euclidean if and only if the matrix $A$ with entries $x_{i j}=d_{0 i}^{2}+d_{0 j}^{2}-d_{i j}^{2}, i, j=1, \ldots, n$ where $d_{i j}$ stands for $d\left(x_{i}, x_{j}\right)$ is positive semi-definite.

We can send $x_{0}$ to the origin of coordinates and in the case that the matrix $A$ is reflexive, we have that

$$
d\left(x_{i}, x_{j}\right)=\sqrt{2} \sqrt{1-x_{i j}} \text { for } i, j=1, \ldots, n .
$$

From this, the next result follows (see also [4]).

Corollary 2.7. Let $s$ be a reflexive similarity on a finite set $X=\left\{x_{1}, \ldots x_{n}\right\}$ with positive semi-definite matrix $S=\left(x_{i j}\right)_{i, j=1, . . n}$ where $x_{i j}$ stands for $s\left(x_{i}, x_{j}\right)$. Then $d: X \times X \rightarrow[0,1]$ defined for all $x_{i}, x_{j} \in X$ by $d\left(x_{i}, x_{j}\right)=\sqrt{1-x_{i j}}$ is a metric and $X$ is isometrically embeddable in an Euclidean space.

It is clear that if a distance $d$ is Euclidean, then $k \cdot d, k>0$ is also Euclidean. Hence, in order to consider euclidianity of distances we can assume that they are valued in $[0,1]$.

The next proposition provides a relationship between distances and Łtransitive reflexive similarities.

Proposition 2.8. [3, 8] Let $s: X \times X \rightarrow[0,1]$ be a reflexive similarity on a set $X . s$ is E-transitive if and only if $1-s$ is a pseudometric on $X$. 
Hence, every distance can be written in the form $1-s$ where $s$ is a reflexive and L-transitive similarity. Therefore, if the conjecture were true, this would say that the square root of any distance would be Euclidean, a fact that contradicts the results in [2].

Indeed, in [2] the authors study the values $c$ for which, given a set $X$ of cardinality $n$ and a distance $d$ on $X$, the power of $d$ to $c\left(d^{c}\right)$ is Euclidean. In particular they prove that for a set of cardinality 6 , the greatest value $c_{6}$ of $c$ is $\frac{1}{2} \log _{2} \frac{3}{2} \sim 0.2924$ which is smaller than $\frac{1}{2}$. Of course, as the cardinality $n$ of the set increases, the corresponding greatest value $c_{n}$ decreases.

Thanks to a result by Blumenthal [1], $c_{4}=\frac{1}{2}$ and the conjecture is true for sets of cardinality smaller than or equal to 4 .

\section{A Reformulation}

In this section we will modify the hypothesis of Conjecture 1.2 in order to obtain a valid result with interesting consequences. For this, we need to recall the definition of continuous Archimedean t-norm [5] and a couple of considerations regarding [2].

Definition 3.1. A continuous Archimedean t-norm $T$ is an operation $T$ : $[0,1] \times[0,1] \rightarrow[0,1]$ such that there exists a continuous decreasing mapping $t:[0,1] \rightarrow[0, \infty]$ with $t(1)=0$ and such that for all $x, y \in[0,1]$

$$
T(x, y)=t^{[-1]}(t(x)+t(y))
$$

where $t^{[-1]}$ is the pseudoinverse of $t$ defined for all $x \in[0,1]$ by

$$
t^{[-1]}(x)= \begin{cases}t^{-1}(x) & \text { if } x \in[0, t(0)] \\ 0 & \text { otherwise }\end{cases}
$$

$t$ is called an additive generator of $T$.

Definition 3.2. [8] Given a set $X$ and a continuous Archimedean t-norm $T$, a similarity $s: X \times X \rightarrow[0,1]$ is $T$-transitive if for all $x, y, z \in X$,

$$
T(s(x, y), s(y, z)) \leq s(x, z) .
$$

The next result relates $T$-transitive similarities with distances. 
Proposition 3.3. [8] Let $X$ be a set, $T$ a continuous Archimedean t-norm and $t$ an additive generator of $T . s: X \times X \rightarrow[0,1]$ a reflexive and $T$ transitive similarity on $X$ if and only if $t \circ s$ is a pseudodistance on $X$.

The next family of continuous Archimedean t-norms (Yager's family) will be useful.

Example 3.4. [5] The Yager's family of continuous Archimedean t-norms $\left(T_{\lambda}\right)_{\lambda \in(0, \infty)}$ is defined for all $x, y \in[0,1]$ by

$$
T_{\lambda}(x, y)=\max \left(\left(1-(1-x)^{\lambda}+(1-y)^{\lambda}\right)^{\frac{1}{\lambda}}, 0\right) .
$$

$t_{\lambda}$ defined by $t_{\lambda}(x)=(1-x)^{\lambda}$ for all $x \in[0,1]$ is an additive generator of $T_{\lambda}$.

N.B.

- If $\lambda>\mu$, then $T_{\lambda}(x, y) \geq T_{\mu}(x, y)$ for all $x, y \in[0,1]$.

- If $\lambda=1$, then we recover the Eukasiewicz t-norm and $t_{1}(x)=1-x$ is an additive generator.

- $\lim _{\lambda \rightarrow \infty} T_{\lambda}(x, y)=\min (x, y)$ for all $x, y \in[0,1]$.

Conjecture 1.2 is not true in its curent forma but now we can state and prove an alternative result.

Proposition 3.5. Let $n$ be a positive integer and $c_{n}$ the greatest value satisfying that for every distance $d$ on any finite set of cardinality $n, d^{c_{n}}$ is an Euclidean distance. Then a reflexive similarity $s: X \times X \rightarrow[0,1]$ on a set $X$ of cardinality $n$ is $T_{\frac{1}{2 c_{n}}}$-transitive if and only if its matrix $S$ is positive semi-definite.

Proof. If $s$ is $T_{\frac{1}{2 c_{n}}}$-transitive, then, thanks to Proposition 3.3, $(1-s)^{\frac{1}{2 c_{n}}}$ is a pseudodistance and by Corollary $2.7(1-s)^{\frac{1}{2 c_{n}} \cdot c_{n}}=(1-s)^{\frac{1}{2}}$ is Euclidean. Hence $S$ is positive semi-definite.

$c_{n}$ is not known except for very few values (for $n=2,3,4,6$, the corresponding $c_{n}$ are $c_{2}=\infty, c_{3}=1, c_{4}=\frac{1}{2}, c_{6}=\frac{1}{2} \log _{2} \frac{3}{2} \sim 0.2924$ [2]) but in [2] a lower bound $k_{n}$ for $c_{n}$ is given. Namely, $k_{n}=\frac{1}{2 n} \log _{2} \mathrm{e} \sim \frac{0.7213}{n}$. Therefore we have the following result 
Proposition 3.6. If a reflexive similarity $s: X \times X \rightarrow[0,1]$ on a set $X$ of cardinality $n$ is $T_{\frac{n}{\log _{2} e}}$-transitive, then its matrix $S$ is positive semi-definite.

In [2] it is conjectured that the value of $c_{n}$ is

$$
c_{n}= \begin{cases}\frac{1}{2} \log _{2}\left(\frac{n}{n-2}\right) & \text { if } n \text { is even } \\ \frac{1}{2} \log _{2}\left(\frac{n^{2}-1}{n^{2}-2 n-1}\right) & \text { if } n \text { is odd. }\end{cases}
$$

From this we can conjecture the following.

\section{Conjecture 3.7.}

- A reflexive similarity $s: X \times X \rightarrow[0,1]$ on a set $X$ of even cardinality $n$ is $T \frac{1}{\log _{2}\left(\frac{n}{n-2}\right)}$-transitive if and only if its matrix $S$ is positive semidefinite.

- A reflexive similarity $s: X \times X \rightarrow[0,1]$ on a set $X$ of odd cardinality $n$ is $T \frac{1}{\log _{2}\left(\frac{n^{2}-1}{n^{2}-2 n-1}\right)}$-transitive if and only if its matrix $S$ is positive semidefinite.

We end this note by showing that Propositions 3.5 and 3.6 provide alternative proofs of two important well known facts.

- Since $\min (x, y) \geq T_{\lambda}(x, y)$ for all $\lambda \in(0, \infty)$ and $x, y \in[0,1]$, every mintransitive and reflexive similarity on a finite set is also $T_{\lambda}$-transitive for all $\lambda \in(0, \infty)$. From Proposition 3.5 it follows the next result (see [6] for an alternative proof).

Proposition 3.8. Every reflexive and min-transitive similarity on a finite set has a positive semi-definite matrix.

- It is well known that $s$ is a reflexive and min-transitive similarity on a set $X$ if and only if $1-s$ is a pseudoultrametric [8]. By the last proposition, its matrix $S$ is positive semi-definite and therefore $\sqrt{1-s}$ is Euclidean. Since the power of pseudoultrametrics are also pseudoultrametrics we obtain a new proof of this well-known result ([10]).

Proposition 3.9. Every ultrametric on a finite set is Euclidean. 


\section{Acknowledgments}

The author thanks M.S. Tomás for thoroughly reading the manuscript and helping to enhance it.

\section{References}

[1] L.M. Blumenthal (1936) Remark concerning the euclidean four-point property. Ergebnisse eines Math. Koll 7 8-10.

[2] M. Deza, H. Maehara (1990) Metric Transforms and Euclidean Embeddings. Transactions of the American Mathematical Society 317 661-671.

[3] S. Gottwald (1992) On t-norms which are related to distances of fuzzy sets. BUSEFAL 50 25-30.

[4] J.C. Gower, P. Legendre (1986) Metric and Euclidean Properties of dissimilarity coefficients. J. Classification 3 5-48.

[5] E. P. Klement, R. Mesiar, E. Pap (2000) Triangular norms, Kluwer, Dordrecht.

[6] B. Moser (2006) On Representing and Generating Kernels by Fuzzy Equivalence Relations. Journal of Machine Learning Research 7 26032620.

[7] R. Nader, A. Bretto, B. Mourad, H. Abbas (2018) On the positive semi-definite property of similarity matrices. Theoretical Computer Science.doi.org/10.1016/j.tcs.2018.06.052.

[8] J Recasens (2011) Indistinguishability Operators. Modelling Fuzzy Equalities and Fuzzy Equivalence Relations. Studies in Fuzziness and Soft Computing. Springer.

[9] I.J. Schoenberg (1938) Metric Spaces and Positive Definite Functions. Transactions of the American Mathematical Society 44 522-536.

[10] I.A. Vestfrid, A.F. Timan (1983) Any separable ultrametric space is isometrically embeddable in $l_{2}$, Funct. Anal. Appl. 17 70-73.

[11] L.A. Zadeh (1965) Fuzzy sets. Information Control 8 338-353. 